\title{
Elektromobilitätsnutzung in Taxiunternehmen und Pflegediensten. Gemeinsamkeiten und Unterschiede der Stakeholderprofile
}

\section{Stakeholder Profiles of Commercial Electric Vehicle Users. Similarities and Differences between Taxi Companies and Care Services}

https://doi.org/10.2478/rara-2020-0030

Eingegangen: 10. Dezember 2019, Angenommen: 5. Oktober 2020

\begin{abstract}
Kurzfassung: Im gewerblichen Bereich ist der Einsatz von Elektrofahrzeugen ökologisch und ökonomisch vielversprechend. Die gezielte Förderung der Nutzung erfordert jedoch eine gute Kenntnis der Nutzergruppen und ihren Anforderungen. Der Beitrag basiert auf einer Untersuchung in Aachen, in der Leitungspersonen von Pflegediensten und Taxiunternehmen befragt wurden, die die Integration von Elektrofahrzeugen erwägen. Erhoben wurden Anforderungen an Elektrofahrzeuge und den Ausbau von Ladeinfrastruktur sowie wahrgenommene Risiken und Potenziale. Die Ergebnisse werden in Stakeholderprofile überführt und um ermittelte Informations- und Partizipationsbedarfe sowie Präferenzen für Formate ergänzt. Es werden große Branchenunterschiede deutlich. Beide Gruppen benötigen branchenspezifisch aufbereitete Informationsangebote. Die Wechselbereitschaft bei Pflegediensten ist höher. Die Fahrzeuge sollen auf dem Betriebsgelände nach den Schichten (meist über Nacht) geladen werden, Schnellladen ist nicht erforderlich. Für den Umstieg werden Praxisberichte und eine Übersicht zu Fördermöglichkeiten benötigt. Die Taxiunternehmen sehen mehr Risiken als Potenziale. Sie benötigen schnell ladende Fahrzeuge (im Zweischichtsystem). Entscheidungsrelevant ist die Bereitstellung von Schnellladeinfrastruktur an Taxiständen. Abstimmungsbedarf zur Konfliktvermeidung betrifft die Positionierung am Taxistand, die Nutzungszeiten sowie die Vermittlung ladender Elektrofahrzeuge.
\end{abstract}

Schlüsselwörter: Nutzung gewerblicher Elektrofahrzeuge, Ladeinfrastruktur, Stakeholderprofil, Nutzeranforderungen, Informationsbedarfe, Pflegedienste, Taxiunternehmen, Aachen

Abstract: The use of electric vehicles in the commercial sector is ecologically and economically promising. To promote the use, however, good knowledge of the (potential) user groups and their needs is required. This paper bases on a survey in Aachen among decision-makers of care services and taxi companies, that are considering the integration of electric vehicles. The requirements for electric vehicles and the construction of charging infrastructure as well as perceived risks and potentials were analysed. The results are transferred to stakeholder profiles. In

\footnotetext{
*Corresponding author: Lorena Niebuhr, RWTH Aachen University, Lehr- und Forschungsgebiet Textlinguistik und Technikkommunikation, Campus-Boulevard 57, 52074 Aachen, Deutschland, E-Mail: I.niebuhr@tk.rwth-aachen.de, ORCID: 0000-0001-6606-5358

Prof. Dr. Eva-Maria Jakobs, RWTH Aachen University, Lehr- und Forschungsgebiet Textlinguistik und Technikkommunikation,

Campus-Boulevard 57, 52074 Aachen, Deutschland, E-Mail: e.m.jakobs@tk.rwth-aachen.de, ORCID: 0000-0002-1407-7979
} 
addition, information and participation requirements were surveyed. The study shows strong sectoral differences. Both groups need sector-specific information offers. The willingness to change is higher in care services. Their vehicles should be charged on the premises after shifts (usually overnight). Fast charging is not necessary. Practice reports and an overview of subsidy opportunities are required. The taxi operators see more risks than potentials. They need fast-charging vehicles (in a two-shift system). The provision of fast-charging infrastructure at taxi stands is relevant for decision-making. Consultation is needed to avoid conflicts concerning the positioning at taxi stands, the times of use and the procurement of charging electric vehicles.

Keywords: Commercial electric vehicle use, Charging infrastructure, Stakeholder profile, User Requirements, Information needs, Care services, Taxi businesses, Aachen

\section{Einleitung}

Deutschland verfolgt das ambitionierte Ziel, durch die Energiewende zur Erreichung von Klimazielen beizutragen. Teil der Energiewende ist die Verkehrswende, das heißt die Umstellung des Verkehrssektors von fossilen Kraftstoffen auf Strom und Kraftstoffe aus erneuerbaren Energien. In diesem Kontext gilt Elektromobilität (im Folgenden E-Mobilität) in Kombination mit Strom aus erneuerbaren Energiequellen als Schlüsseltechnologie. Der Handlungsbedarf des Umdenkens ist angesichts verkehrsbedingt hoher Luftverschmutzung und $\mathrm{CO}_{2}$-Emmissionen hoch, die Bereitschaft, auf Elektrofahrzeuge (im Folgenden E-Fahrzeuge) umzusteigen, jedoch bislang gering. Ein vielversprechender Einsatzbereich ist die gewerbliche Nutzung. Der Anteil gewerblich genutzter Fahrzeuge (rund 5 Mio.) ist im Vergleich zu privat genutzten (rund 42 Mio.) gering, aber knapp zwei Drittel der jährlichen Neuzulassungen in Deutschland (65,5 \%) erfolgen für gewerbliche Nutzer (Kraftfahrt-Bundesamt 2020). Der Anteil des Wirtschaftsverkehrs am Stadtverkehr beträgt mehr als ein Drittel (etwa 35-50 \%) (Kampker/Deutskens/ Maue et al. 2016: 299; NOW 2018: 3).

Studien zeigen, dass je nach Nutzungsszenario ein Großteil von Flottenfahrzeugen durch E-Fahrzeuge ersetzt werden kann, ohne Abläufe zu stören oder signifikante Mehrkosten bzw. Gewinneinbußen zu erzeugen (z. B. Goldschmidt/Richter/Pfeil 2019: 6). Insbesondere Anwendungsfälle mit hohen Jahresfahrleistungen haben wirtschaftliches Potenzial (Kaplan/Gruber/Reinthaler et al. 2016: 12).

Der mit der Verkehrswende intendierte soziotechnische Transformationsprozess muss von den Beteiligten getragen werden (vgl. Becker/Renn 2019). Dies ist bislang nur bedingt der Fall. Trotz inrer Potenziale ist die Diffusion von E-Fahrzeugen gering. Das Ziel der Bundesregierung, eine Million E-Fahrzeuge bis 2020 einzuführen, musste auf 2022 verschoben werden. Ein Schwachpunkt ist das Fehlen von Ladeinfrastruktur
(Grausam/Parzinger/Müller 2015: 31). Diesen Aspekt adressiert das Verbundprojekt „ALigN - Ausbau von Ladeinfrastruktur durch gezielte Netzunterstützung"." Ziel des Projektes ist der massive lokale Ausbau der Ladeinfrastruktur in Aachen (900 Ladepunkte). Der Ausbau erfolgt für gewerbliche Nutzer bzw. Unternehmen, die bereit sind, auf E-Mobilität umzusteigen bzw. diese stärker zu nutzen. Die Entwicklung passgenauer Lösungen, die der Unterschiedlichkeit daraus resultierender Zielgruppen gerecht wird, erfordert ein gutes Verständnis ihrer Bedarfe in puncto Ladeinfrastruktur wie auch potenzieller Kommunikationsbedarfe bei der Umstellung auf E-Mobilität. Dieser Beitrag ist Teil der Begleitforschung, die Aussagen dazu intendiert. Der Beitrag stellt Ergebnisse einer qualitativen Studie zu Pflegediensten und Taxiunternehmen vor. In der Studie wurden Entscheider und Entscheiderinnen in Unternehmen (hier Stakeholder) $(n=12)$ befragt, die die Anschaffung eines E-Fahrzeugs erwägen, den Kauf aber von der Sachlage abhängig machen, z. B. der Ladeinfrastruktur in ihrem Geschäftsumfeld. Der Fokus der Studie richtet sich auf drei Fragen:

- Welche organisationalen Merkmale der Zielgruppen beeinflussen die Nutzung von E-Fahrzeugen und Ladeinfrastruktur?

- Welche Potenziale und Risiken werden bezogen auf den Einsatz von E-Fahrzeugen wahrgenommen?

- Welche Anforderungen stellen die Zielgruppen an E-Fahrzeuge, Ladeinfrastruktur und Informationsangebote für die Umstellung auf E-Mobilität?

Ausgehend von einem Literaturüberblick zur gewerblichen Nutzung von E-Mobilität (Kapitel 2.1), insbesondere durch Pflegedienste (Kapitel 2.2) und Taxiunternehmen (Kapitel 2.3), werden Ansätze zur Bestimmung von Stakeholderprofilen vorgestellt (Kapitel 2.4) sowie

1 https://www.acs.eonerc.rwth-aachen.de/go/id/sbcq (25.08.2020). 
Aspekte der Akzeptanz von Kommunikation von E-Mobilität thematisiert (Kapitel 2.5). Kapitel 3 gibt einen Überblick über die Methodik der Untersuchung. In Kapitel 4 werden die Stichproben bezogen auf Firmenmerkmale charakterisiert (Kapitel 4.1) sowie branchenbezogene Einstellungen zu E-Mobilität (Kapitel 4.2), Anforderungen an E-Fahrzeuge und Ladeinfrastruktur für die Nutzung in Flotten (Kapitel 4.3) sowie Informations- und Partizipationsbedarfe (Kapitel 4.4) beschrieben. Die Ergebnisse werden abschließend (Kapitel 5) kritisch diskutiert und es wird Handlungsbedarf für Forschung und Praxis abgeleitet.

\section{Stand der Forschung}

\subsection{Gewerbliche Nutzung von E-Mobilität}

Mit dem anhaltenden Zuzug von Menschen und Unternehmen in die städtischen Räume steigt dort der Anteil des gewerblichen Verkehrs (Witte/Klumpp/Keuschen et al. 2013: 46). Unternehmen, die eine Flotte unterhalten, nutzen ihre Fahrzeuge dabei für interne oder externe Dienstleistungen in den Bereichen Güter- oder Personenverkehr (Gnann/Plötz/Funke et al. 2015). Der Einsatz von E-Fahrzeugflotten hat aufgrund der im Vergleich zu privaten Nutzungskontexten hohen Laufleistung ein großes ökologisches und ökonomisches Potenzial. Flottenmerkmale wie vorab geplante Strecken, sich wiederholende Fahrprofile und der regelmäßige Austausch der Fahrzeuge sind ideal für E-Fahrzeuge (Ketelaer/ Kaschub/Jochem et al. 2014). Insbesondere die hohe Laufleistung bei gleichzeitig niedrigen Betriebskosten ermöglicht eine schnelle Amortisierung bei gewerblichen Anwendungen und damit die Kompensation der aktuell höheren Initialkosten von E-Fahrzeugen im Vergleich zu konventionellen Fahrzeugen (Gnann/Plötz/Funke et al. 2015: 183). Geplante Strecken und sich wiederholende Fahrprofile erlauben, Ladeinfrastruktur an festen Standorten zu nutzen (Ketelaer/Kaschub/Jochem et al. 2014: 2170). Im Schichtbetrieb haben Fahrzeuge meist keine lange Standzeit. Dies reduziert die Zeitfenster für das Laden oder erfordert einen erhöhten Planungsaufwand.

\subsection{Nutzung von E-Mobilität durch Pflegedienste}

Die ambulante Pflege ist ein stetig und schnell wachsender Bereich. Treiber sind die Alterung der Bevölkerung und der Wunsch älterer Menschen, in ihrer gewohnten Umgebung zu bleiben (vgl. Kis/Augustin/Augustin 2017). Pfleger/-innen besuchen ihre Patientinnen/Patienten regelmäßig, teilweise mehrmals täglich. Für ihre Touren, die im Voraus geplant werden, nutzen sie Kleinstwagen (Uniper Technologies 2016: 40). Die Routen wiederholen sich regelmäßig (Vogel 2016: 6). Die Laufleistung pro Schicht ist überschaubar: Es gibt hohe Standzeiten der Fahrzeuge zwischen den Fahrten, die zum Laden genutzt werden können und Hinweise darauf, dass die jährliche Laufleistung höher ist als bei Privatfahrzeugen (Plötz/Gnann/Ullrich et al. 2014: 13). Dies spricht für den Einsatz von E-Mobilität.

Die Anforderungen von Pflegediensten an E-Mobilität ist Gegenstand verschiedener Forschungsprojekte in Deutschland, z. B. des Projekts GO ELK! (Uniper Technologies 2016), das den Einsatz von E-Fahrzeugen in gewerblichen Kleinflotten unter anderem mit dem Ziel begleitete, ein exemplarisches Ladeprofil für Pflegedienste zu erstellen. Das ermittelte Profil zeigt, dass die Flottenfahrzeuge auf dem Firmengelände geladen werden, hauptsächlich über Nacht. Damit entfällt die Nutzung der (halb)öffentlichen Ladeinfrastruktur. Aufgrund der geringen Laufleistung pro Schicht kann es auch ausreichend sein, nur einmal pro Tag zu laden. Werden E-Fahrzeuge in einem Zweischichtsystem eingesetzt, kann eine zusätzliche Zwischenladung zwischen den Schichten erforderlich sein.

Das Projekt „Smart E-User“ (Ehrler/Luft/Elektirikçi et al. 2016) betrachtet weitere Merkmale der E-Fahrzeugnutzung unter anderem in Pflegediensten, wie den Einfluss der jahreszeitlichen Witterung auf Ladezeiten. Die Ergebnisse bestätigen die oben genannten Befunde. Bevorzugt wird auf dem Betriebsgelände zwischen den Schichten oder in Pausen geladen. Die Nutzung der öffentlichen Ladeinfrastruktur während einer Tour wird abgelehnt. Wenn Mitarbeiter/-innen Flottenfahrzeuge mit nach Hause nehmen, benötigen sie dort eine Ladeoption sowie ein Abrechnungssystem für den Stromverbrauch oder müssen das E-Fahrzeug an einer öffentlichen Ladesäule laden und dies abrechnen.

Grausam, Parzinger und Müller (2015) begleiteten die Integration von E-Fahrzeugen in öffentlich und privatwirtschaftlich betriebenen Flotten (u. a. Pflegedienste) in unterschiedlichen deutschen Städten. Es wurden die Nutzungsakzeptanz von E-Fahrzeugen, nutzerspezifische Motive und die Rahmenbedingungen für einen erfolgreichen Betrieb erörtert. Die Akzeptanz von E-Fahrzeugen ist im Flottenmanagement hoch. Die befragten Pflegedienste planten, in Zukunft ein Drittel der Flotte auf E-Fahrzeuge umzustellen. Hauptmotive 
des Wechsels sind niedrigere Kosten im Vergleich zu herkömmlichen Fahrzeugen und das Anwerben junger Mitarbeiter/-innen durch ein umweltfreundliches, modernes Image. Die Fahrzeuge werden auf dem Betriebsgelände geladen. Im ambulanten Zweischichtsystem müssen die Pflegedienste schnellladen. Die Installation eigener Ladeinfrastruktur sollte (rechtlich) einfacher und kostengünstiger möglich sein. Der Antrags- und Installationsprozess sollte standardisiert werden.

Als Fazit kann festgehalten werden, dass der Einsatz von E-Fahrzeugen in ambulanten Pflegediensten möglich und vielversprechend ist. Als notwendige Voraussetzung gilt eine Ladestruktur, die auf dem Betriebsgelände verfügbar ist und Schnellladen (je nach Arbeitszeitgestaltung) zulässt. Die vom Unternehmen gestattete private Nutzung von Flottenfahrzeugen setzt die Möglichkeit des Ladens am Wohnort sowie ein Abrechnungssystem voraus, das diese Form der Nutzung erfasst.

\subsection{Nutzung von E-Mobilität durch Taxiunternehmen}

In vielen Städten der Welt nutzen Taxiflotten E-Fahrzeuge. Die Größe der Taxiunternehmen variiert abhängig von den lokalen Gegebenheiten von Klein- bis Großunternehmen. Teilweise sind Taxiunternehmen Einpersonenbetriebe, die Taxiunternehmer/-innen fahren selbst. Taxiunternehmen sind meist in Verbänden organisiert. Die Verbände regeln die Verteilung der Fahrten und vertreten die Interessen der Mitglieder. In Deutschland kann die Kundschaft eine Taxifahrt initiieren, indem sie ein Taxi an einem Taxistand nimmt oder ein Taxi telefonisch ordert. Am Ende einer Kundenfahrt entscheidet der Taxifahrer bzw. die Taxifahrerin, ob eine neue Fahrt per Taxifunk angenommen, zum Betriebsgelände gefahren wird oder zu einem Taxistand, um dort auf Kundschaft zu warten (§ 47 Abs. 1 PBefG). ${ }^{2}$ Per GPS-Tracking wird die Position erfasst, außerdem werden in den Taxis Lenk-, Warte- und Pausenzeiten erfasst (Zhang/Sun/Li et al. 2015).

Es gibt viele Studien, die die Elektrifizierung von Taxiflotten simulieren (u. a. Tian/Jung/Wang et al. 2016; $\mathrm{Ko} / \mathrm{Kim} / \mathrm{Nam}$ et al. 2017; Wang/Zhang/Fang et al. 2020). Ziele der Simulationen sind unter anderem Aussagen zum Ladebedarf, zur Platzierung von Ladeinfrastruktur oder zur Verbesserung des Lademanagements. Nach der Simulationsstudie von Sellmair und Hamacher

2 Personenbeförderungsgesetz in der Fassung der Bekanntmachung vom 8. August 1990, das zuletzt durch Artikel 329 der Verordnung vom 19. Juni 2020 geändert worden ist.
(2014) ist der Einsatz von E-Fahrzeugen im Taxibetrieb geeignet, aber aufgrund hoher Fahrleistungen problematisch. Für den Einsatz spricht das Fahrprofil - regelmäßige Fahrten und kurze Pausen, in denen Fahrerin oder Fahrer auf die nächste Kundschaft wartet. Gegen die Nutzung spricht eine hohe tägliche Laufleistung. Als bester Ort des Aufladens werden Taxistände identifiziert. Dort sollte eine schnellladefähige Ladeinfrastruktur installiert werden.

Grausam, Parzinger und Müller (2015: 159) befragten Taxiunternehmer/-innen, die E-Fahrzeuge einsetzen, und ihre Fahrerinnen und Fahrer zum Umstieg und zur Nutzung von E-Fahrzeugen. Zu Projektbeginn war die Akzeptanzbereitschaft der Taxiunternehmer/ -innen und Taxifahrer/-innen gegenüber E-Taxis gering, sie nahm jedoch mit der Zeit zu. Die öffentliche Infrastruktur wurde gelegentlich zum Zwischenladen genutzt, die meiste Zeit wurden die E-Fahrzeuge über Nacht, am eigenen Betriebsgelände, geladen. Als wesentliche Bedarfe werden ein Auftragsvergabesystem, das Kundinnen und Kunden erlaubt, gezielt E-Taxis anzufordern, genannt, darüber hinaus der Ausbau der öffentlichen Ladeinfrastruktur (Schnellladen), taxi-eigene (Schnell-) Ladeinfrastruktur (vornehmlich an Taxiständen) sowie das Aufrücken mit herkömmlichen Taxis trotz Ladens am Taxistand (wie auch der Wunsch nach Weiterentwicklung der Induktivladetechnik).

Im Projekt „GuEST“ wurden Stuttgarter Taxiunternehmen mit E-Fahrzeugen ausgestattet und finanzielle Verluste kompensiert. Der Feldversuch wurde mit Fokusgruppen und Befragungen begleitet (Goldschmidt/ Richter/Pfeil 2019). Die teilnehmenden Taxiunternehmen wurden charakterisiert und erfolgsrelevante Rahmenbedingungen identifiziert. Die organisatorisch-strategischen Unterschiede zwischen den Taxiunternehmen beeinflussen ihre Aktivität und Wahrnehmungen. Der Entscheidungsfindungsprozess für die Umstellung auf E-Fahrzeuge ist ein Abwägen ökologischer und ökonomischer Aspekte. Zentrale Motive des Einsatzes von E-Fahrzeugen sind ihr Beitrag zur Umweltfreundlichkeit des Unternehmens und zur Lebensqualität in der Heimatstadt sowie das Testen einer Innovation. Auf der administrativen Ebene wurde auf Bestreben der Taxiunternehmen ein günstiger Ladestromtarif ausgehandelt, ohne den der Betrieb wirtschaftlich nicht möglich wäre. Als zentrale technische Entwicklungsaufgabe sehen Fahrpersonal und Taxiunternehmen die Harmonisierung von Reichweite und Ladeinfrastruktur. Ein wichtiger Faktor ist der Einfluss der Witterung auf den E-Taxibetrieb; im Winter sinkt die Reichweite, der Einsatz wird aufwendiger. 
Helmus und van den Hoed (2015) analysierten das Ladeverhalten gewerblicher E-Fahrzeugnutzer/-innen in Amsterdam. Die Autoren befragten Behörden der Stadt, leiteten Nutzergruppen ab und erhoben nutzergruppenspezifische Daten zu Ladevorgängen. Die Ergebnisse zeigen, dass E-Taxifahrer/-innen Zeit und Ort des Ladens freier wählen können als andere gewerbliche Nutzer/-innen, die stärker an Spezifikationen gebunden sind. Das Ladeverhalten ist heterogen. Geladen wird zwischen den Schichten oder über Nacht an der öffentlichen Ladeinfrastruktur in der Nähe ihres Hauses, bei größeren Taxiunternehmen in Pausen auf dem Betriebsgelände oder bei organisatorischen Aufgaben. Während der Schicht nutzen die Befragten die öffentliche Ladeinfrastruktur in der ganzen Stadt. Tesla-Nutzer/-innen präferieren die Tesla-eigene Ladeinfrastruktur. Das Ladeprofil ist sehr heterogen und dennoch über Wochen konstant, da die Unterschiede zwischen Wochentagen und Wochenenden gering sind.

Asamer, Reinthaler, Ruthmair et al. (2016) betrachten den Einsatz von E-Taxis in Wien. Ein zentrales Ergebnis der Studie ist die insgesamt geringe Akzeptanz von E-Taxis bei Taxifahrer/-innen. Das Laden während des Wartens auf Kundschaft und der Einsatz von E-Taxis im Zweischichtsystem erfordern eine schnelle Aufladung. Die Taxifahrer/-innen wünschen sich Batteriewechselstationen, um Wartezeiten für Ladevorgänge minimieren zu können.

Die Begleitung von E-Taxiflotten zeigt, dass der Einsatz von E-Fahrzeugen stark durch organisationale Merkmale geprägt wird. Taxiunternehmen stellen spezielle Anforderungen an die technischen und organisationalen Rahmenbedingungen und weisen eine insgesamt geringe Bereitschaft auf, E-Fahrzeuge in ihre Flotte zu integrieren.

\subsection{Stakeholderprofile für den Ausbau der Ladeinfrastruktur}

Infrastrukturprojekte, hier zum Ausbau der Ladeinfrastruktur, erfordern aussagekräftige Beschreibungen potenzieller Stakeholder (Jakobs 2019). Sie sind Voraussetzung für ein effizientes Stakeholdermanagement, das Vermeiden von (Interessen)konflikten und das effiziente Einbeziehen von Stakeholdergruppen (Krips 2017: 6). Stakeholderprofile können literaturbasiert oder empirisch erarbeitet werden, z. B. durch Zielgruppenbefragung, Interviews mit Personen, die die Zielgruppen kennen (Helmus/van den Hoed 2015), oder anderen Methoden (vgl. Jakobs 2019). Die Darstellung der Profile kann textuell erfolgen oder visuell gestützt. Eine Variante, die hier aufgegriffen wird, ist die Darstellung und der Vergleich von Stakeholderprofilen über Karten (Gölz/ Langer/Becker et al. 2019: 92). Die Autoren erheben anhand qualitativer Leitfadeninterviews mit Stakeholdern (Lokalpolitiker/-innen, Verwaltungsangehörige, Bürger/ -innen, Vertreter/-innen des lokalen Tourismusverbands; $\mathrm{n}=19$ ) deren Sicht auf lokale Maßnahmen eines Mobilitätsprojekts. Aus den Daten werden Profile abgeleitet, die den Projektbeteiligten ein vertieftes Verständnis der sozialen Wahrnehmung (Repräsentation) des Projekts und seiner Gegenstände durch die Akteure ermöglichen sollen. Die Grundannahme ist, dass Wahrnehmung und Akzeptanz regional geprägt sind.

Die Rekonstruktion der sozialen Projekt-Repräsentation berücksichtigt die Verortung der Befragten in der Region, ihre Kenntnis zentraler Entwicklungslinien der Gemeinde, ihre Sicht auf die Energie- und Mobilitätswende, auf Chancen und Risiken (für die Region, für sie selbst) sowie wahrgenommene Treiber und Hemmnisse des Projekts. Teil sozialer Repräsentationen sind (gesellschaftliche) Normen, Ortsverbundenheit, ein kollektives Gedächtnis und geteiltes gruppenspezifisches Wissen. Der Vergleich gruppenbezogener Repräsentationen erlaubt die Ermittlung von Gemeinsamkeiten und Unterschieden. Die Darstellung erfolgt in Form einer Landkarte der sozialen (Projekt-)Repräsentation. Diese umfasst die wahrgenommenen Chancen und Treiber, Risiken und Hemmnisse. Die Karten zeigen in einer tabellarischen Übersicht mit unterschiedlicher Zellenmarkierung, wie stark ein Item wahrgenommen wird. Der Ansatz erlaubt, Unterschiede schnell erfassbar darzustellen, Einzelinformationen zu verdichten, Themen zu gewichten und für Unterschiede zu sensibilisieren.

\subsection{Akzeptanz und Kommunikation von E-Mobilität}

In Studien zur gewerblichen Nutzung von E-Fahrzeugen wird mehrfach das Thema Akzeptanz angesprochen. Wie eingangs erwähnt, ist die Akzeptanz von E-Mobilität eher gering, wenn mit Akzeptanz aktives Engagement (Becker/Renn 2019) gemeint ist, das sich in Verhaltensänderungen zeigt (Wechsel zu oder Nutzung von E-Fahrzeugen). Als Akzeptanzhemmer werden Unsicherheit bei der Reichweite sowie fehlende Ladeinfrastruktur genannt (Grausam/Parzinger/Müller 2015: 135), positiv wahrgenommen wird die finanzielle Förderung von E-Fahrzeugen und Ladeinfrastruktur (Globisch/ Dütschke/Wietschel 2018: 122). Aktive Akzeptanz und 
die Adaption der Technologie der E-Mobilität erfordern weitere Maßnahmen, $z$. B. Informationskampagnen und aktives Einbeziehen der Akteure (Goldschmidt/Richter/ Pfeil 2019: 2). In diesem Beitrag wird Akzeptanz als Ergebnis von Bewertungsprozessen gesehen, die technologie-, kontext- sowie gruppen- bzw. individuenbezogen unterschiedlich verlaufen (Jakobs 2019).

Kommunikation gilt als notwendige, aber nicht hinreichende Bedingung für aktive Akzeptanz (Renn 2015). Nichtwissen wie auch Wissen können zu aktiver Ablehnung oder Unterstützung führen (Bozem/Nagl/Rath et al. 2013: 33). Zu den Informations- und Partizipationsbedarfen für den potenziellen Umstieg auf Elektromobilität gibt es bisher kaum Studien. Dumortier, Siddiki, Carley et al. (2015) betrachten den Zusammenhang von Information und Akzeptanz. Ihre Ergebnisse zeigen einen positiven Effekt von „Total Cost of Ownership“-Informationen ${ }^{3}$ auf die Entscheidung zur Nutzung von E-Fahrzeugen. Krause, Carley, Bradley et al. (2013) erheben Wissen über und die Wahrnehmung von Elektromobilität in den USA. Die Studie zeigt große Wissenslücken sowie falsche Annahmen seitens der Teilnehmenden zum Thema. Falsche Annahmen zu Kaufpreis, Fördermitteln und Einsparungen beim Unterhalt eines E-Fahrzeugs beeinflussen signifikant die Kauf- und Nutzungsabsichten der Befragten.

Die Entwicklung von Kommunikationsangeboten für Stakeholder, die den Wechsel zu Elektromobilität erwägen, erfordert eine gute Kenntnis ihrer Bedarfe und Themen sowie der Faktoren, die Einfluss auf ihre Entscheidungen haben (Reimer/Jakobs/Borg et al. 2015; Hellmuth/Jakobs 2019). Im gewerblichen Bereich hängt die Entscheidung über die Anschaffung von E-Fahrzeugen von organisationalen Merkmalen ab wie auch von den persönlichen Ansichten der dafür zuständigen Person (Kaplan/Gruber/Reinthaler et al. 2016; Globisch/ Dütschke/Wietschel 2018). Argumentationen für diese Zielgruppe müssen beide Aspekte berücksichtigen.

Meinungsbildung entsteht oft im Austausch, das heißt in der Interaktion mit anderen. Als wichtig gilt unter anderem die Möglichkeit der Partizipation, das heißt der aktiven, freiwilligen Beteiligung von Akteuren an Gestaltungs- und Entscheidungsprozessen (vgl. Nanz/Fritsche 2012; Goldschmidt/Richter/Pfeil 2019). Im Forschungs-

3 Als Total Cost of Ownership werden die Gesamtnutzungskosten bezogen auf die Lebens- oder Nutzungsdauer bezeichnet. Im Fall von Fahrzeugen beinhaltet die Betrachtung beispielsweise Kaufpreis, Kraftstoffkosten, weitere Betriebskosten und gegebenenfalls auch den Wiederverkaufswert (Dumortier/Siddiki/Carley et al. 2015: 72). projekt „Online Schaufenster Elektromobilität" wurden verschiedene Bürgerbeteiligungsinstrumente entwickelt, die von den Zielgruppen positiv bewertet wurden (Fröschle 2017: 509). Dazu gehören unter anderem eine zentrale Webplattform, eine mobile App (LadestationSuche), ein Beteiligungsspiel (Welche Mobilitätsform passt zu mir?) sowie Social-Media-Kanäle zu dem Projekt.

An dieser Stelle kann festgehalten werden, dass es bisher kaum Studien zu den Informations- und Partizipationsbedarfen gewerblicher Flottennutzer gibt, die den Wechsel zu Elektromobilität erwägen oder planen. Die wenigen vorliegenden Studien zeigen, dass mangelndes Wissen und falsche Annahmen die Nutzungsbereitschaft senken. Insgesamt gibt es Bedarf nach Informationsund Beteiligungsformaten.

\section{Methodik}

Die diesem Beitrag zugrunde liegende Untersuchung ist qualitativ-explorativ angelegt. Die Datenerhebung erfolgt mit leitfadenbasierten Tiefeninterviews. Diese Methode ermöglicht es, intensiv nachzufragen und so auch Themen zu erfassen, die bei der literaturbasierten Entwicklung des Leitfadens nicht gesehen wurden. Die Zielgruppenauswahl orientiert sich am zielgruppenspezifischen Potenzial für Elektromobilität.

Die Akquise orientierte sich an zwei Kriterien: Voraussetzung war zunächst, dass das entsprechende Unternehmen bereit ist, Elektrofahrzeuge in seinen Fuhrpark zu integrieren. Anschließend wurden die dafür im Unternehmen zuständigen oder weisungsberechtigten Personen (Fuhrparkverantwortliche bzw. Unternehmensleitung befragt (vgl. Kaplan/Gruber/Reinthaler et al. 2016). Insgesamt wurden 26 Pflegedienste kontaktiert, die in Aachen Dienste anbieten; davon erfüllten acht Unternehmen die erste Voraussetzung. Von diesen acht Unternehmen waren sechs zuständige oder weisungsbefugte Personen bereit zu einem Interview. Die Zielgruppe Taxiunternehmen wurde mit verschiedenen Mitteln adressiert, wie Vermittlung durch die Industrie- und Handelskammer, Telefonate mit großen Taxizentralen, Anrufe bei Taxiunternehmen, Akquise über einen lokalen Autohändler mit speziellen Verkaufsprogrammen für Taxiunternehmen sowie persönliches Ansprechen an Taxiständen. Sechs Vertreter/-innen von Taxiunternehmen, die die erste Voraussetzung erfüllten, waren zu einem Interview bereit. Die Gesprächsbereitschaft war generell gering. Nichtbereitschaft gründete auf dem Fehlen einer finanziellen Vergütung oder Sprachbarrieren. 
Die Datenerhebung erfolgte 2019 in Aachen. Die Probanden $(n=12)$ erhielten mehrheitlich vorab den Interviewleitfaden. Die Interviews wurden meist am Arbeitsplatz geführt. Der Leitfaden umfasst sechs Themenkomplexe:

- Einstellung zu E-Mobilität, zukünftige Nutzung von E-Fahrzeugen im Unternehmen

- Anforderungen an E-Fahrzeuge

- Anforderungen an den Ladeinfrastrukturausbau und Standorte

- Präferenzen für Ladeszenarien

- Installation der Ladeinfrastruktur auf dem Betriebsgelände

- Informationsbedarfe

Ergänzend wurden Angaben zu Unternehmensmerkmalen, zum Fuhrpark und zur Arbeitsorganisation erhoben sowie soziodemographische Daten der Befragten (das Alter wurde geschätzt), ihr Interesse an E-Mobilität und ihre Einschätzung der Relevanz geförderter E-Mobilität in ihrem Geschäftsfeld. In einem Screeningbogen wurden arbeitsorganisatorische und sozioökonomische Aspekte des Unternehmens erhoben. Die Entwicklung berücksichtigt Interessen der Stakeholdergruppen im regionalen Kontext, Motive für Entscheidungen, (Unternehmens-)Merkmale, Verhaltensweisen, Anforderungen, Präferenzen sowie wahrgenommene Potenziale und Risiken (Krips 2017: 13). Die Interviews wurden als Audiofile aufgezeichnet (durchschnittliche Länge: 38 Minuten), transkribiert (Leseversion) und anonymisiert ( $P$ = Pflegedienste, $T$ = Taxiunternehmen).

Die Befragten in den Pflegediensten $(n=6)$ haben eine Führungsposition inne oder sind für den Fuhrpark verantwortlich. In kleinen Pflegediensten übernehmen die Fuhrparkverantwortlichen auch Pflegeaufgaben. Die Befragten (vier männlich, zwei weiblich) sind im Durchschnitt 54 Jahre alt (geschätzt). Ihre durchschnittliche Berufserfahrung in der Pflegebranche beträgt 28,7 Jahre. Drei Befragte waren vorher in anderen Bereichen des Gesundheitswesens tätig, zwei in der Verwaltung von Unternehmen, eine Person im Qualitätsmanagement einer Softwarefirma. Niemand von innen nutzt privat ein E-Fahrzeug.

Es wurden fünf Taxiunternehmer und eine Taxiunternehmerin befragt. Zwei koordinieren ihr Unternehmen inklusive Fahrer, Fahrerinnen und Fahrzeuge; vier Befragte fahren zusätzlich selbst. Das durchschnittliche Alter liegt bei 54 Jahren (geschätzt). Drei Befragte geben an, „schon immer" in der Taxibranche gearbeitet zu haben, einer davon ohne Berufsausbildung, zwei weitere seit dem Abschluss ihres Studiums. Ein Befrag- ter war in der Elektronikproduktion beschäftigt, bevor er sich mit einem Taxiunternehmen selbstständig machte, ein anderer arbeitete früher als Monteur, ein weiterer im Maschinenbau. Die Berufserfahrung der Befragten in der Taxibranche beträgt im Durchschnitt 25,2 Jahre. Keiner der Befragten nutzt privat ein extern ladbares E-Fahrzeug, wohingegen zwei Befragte Vollhybride nutzen.

Die Datenanalyse erfolgte qualitativ inhaltsanalytisch nach Mayring (2015) in MAXQDA. Das deduktiv wie induktiv gebildete Kategoriensystem umfasst acht Ober-, 40 Sub- und 253 Subsubkategorien. Die Analyse erfolgte per Stakeholdergruppe sowie im Vergleich. Die Ergebnisdarstellung wird durch typische Nutzerkommentare illustriert. Die Ergebnisse wurden in Anlehnung an Gölz, Langer, Becker et al. (2019) in Profilkarten erfasst. In den Karten werden links Items gelistet, rechts wird die Relevanz dieser Punkte per Stakeholdergruppe angezeigt, gemessen an der Anzahl von Nennungen (dunkelgrau: relevant, Item wird von ein bis drei Personen genannt; dunkelgrau mit Punkt: hoch relevant, Item wird von vier und mehr Personen angesprochen; weiß: keine Relevanz).

\section{Ergebnisse}

\subsection{Unternehmensmerkmale}

\section{Pflegedienste}

Der Personalstand in den befragten Unternehmen $(n=6)$ variiert zwischen 35 bis 90 Personen. Die Flotten umfassen fünf bis 160 Fahrzeuge. Der Pflegedienst mit 160 Fahrzeugen operiert überregional. Die Geschäftsräume der Pflegedienste sind angemietet (vgl. Grausam/Parzinger/Müller 2015: 126). Alle Pflegedienste arbeiten im Zweischichtsystem (Früh- und Spätschicht), einer im Notfall auch im Dreischichtsystem. Die Fahrzeiten variieren je nach Route und Pflegeaufwand pro Patient bzw. Patientin. Sie beschränken sich auf das Aachener Stadtgebiet. In einem Fall werden gelegentlich auch Personen in Nachbargemeinden versorgt. Die durchschnittlich pro Schicht zurückgelegte Strecke beträgt $42,5 \mathrm{~km}$ (vgl. Uniper Technologies 2016: 66). Bei zwei Pflegediensten können nicht alle Fahrzeuge auf dem Betriebsgelände geparkt werden, hier wird partiell ein öffentlicher Parkplatz genutzt.

\section{Taxiunternehmen}

Die Größe der Unternehmen ( $n=6)$ reicht vom Ein-MannUnternehmen bis zu Unternehmen mit zehn Fahrerin- 
nen und Fahrern (vgl. Goldschmidt/Richter/Pfeil 2019). Je nach Mitarbeiteranzahl variiert die Anzahl der Fahrzeuge zwischen einem und sechs Fahrzeugen. Hauptzweck der Fahrzeugnutzung ist der Personen- und gelegentlich der Warentransport. Bis auf einen selbstständigen Taxifahrer, der nur tagsüber arbeitet, operieren alle befragten Taxiunternehmen im Zweischichtsystem mit Tag-Nacht-Wechsel. Die Fahrten können in der Regel nicht vorab geplant werden. Der Einzugsbereich umfasst das Stadtgebiet und die umliegenden Gemeinden. Standortspezifisch sind gelegentliche (über)regionale Fahrten zu umliegenden Flughäfen oder Städten in einer Entfernung von etwa 100-200 km. Wenn öffentliche Verkehrsmittel ausfallen, müssen weit(er) entfernte Ziele im europäischen Ausland angefahren werden. In Tagesschichten beträgt die zurückgelegte Strecke im Mittel 176,67 km (vgl. Grausam/Parzinger/Müller 2015: 159). Bei Nachtschichten ist sie tendenziell geringer $(168,33$ $\mathrm{km})$. Die Ausgangspunkte der Touren differieren stark. Ein Befragter nennt die Firmenzentrale seines Unternehmens, ein anderer Befragter gibt an, dass die Fahrzeuge auf seinem Privatgelände, öffentlichen Parkplätzen sowie einem gemieteten Standplatz in der Innenstadt geparkt sind, wo sie von seinen Fahrerinnen/Fahrern abgeholt und nach der Schicht zurückgebracht werden. Ein anderer selbstständiger Taxifahrer parkt das Fahrzeug auf seinem Privatgelände oder in häuslicher Nähe auf einem öffentlichen Parkplatz. Alle Taxiunternehmen, die Geschäftsräume unterhalten, haben diese gemietet oder nutzen entsprechend deklarierte Teile ihrer Wohnung. Die Geschäftsräume und Privathäuser bieten keine E-Ladeinfrastruktur.

\subsection{Einstellung zur E-Mobilität}

\section{Pflegedienste $(P)$}

Die Befragten schätzen den Beitrag gewerblich genutzter Elektromobilität zum Umweltschutz. Das positive Image von E-Fahrzeugen trage zur Arbeitgeberattraktivität bei (vgl. Grausam/Parzinger/Müller 2015). „Ich denke, dass der Dienstwagen attraktiver wird und ich glaube, dass sich unsere Mitarbeiter mit diesem Trend identifizieren und daran glauben. [...] Lassen Sie es mich richtig formulieren: Es ist ja kein Trend. Sie finden es gut, sich aktiv an Elektromobilität zu beteiligen" (Interview P4).

Positiv bewertet wird der höhere Komfort von E-Fahrzeugen. Zwei Befragte erwarten einen stärker vorausschauenden Fahrstil und den Abbau von Konflikten in der Belegschaft, die entstehen, wenn konventionelle Fahrzeuge von ihren Fahrerinnen/Fahrern nach der Nutzung nicht betankt wurden. Durch den Einsatz von E-Fahrzeugen und ihr Laden direkt nach der Rückkehr in die Pflegeeinrichtungen könnten diese Konflikte vermieden werden. Das letztendlich wahrgenommene Potenzial sind Kosteneinsparungen (vgl. Grausam/Parzinger/Müller 2015). Die Befragten sehen das Potenzial, im Einsatz Kosten zu sparen. Die Initialkosten beim Umstieg auf Elektromobilität werden als zu hoch angesehen, jedoch nicht als kritisch, da es finanzielle Spielräume für Investitionen gäbe (vgl. aber Grausam/ Parzinger/Müller 2015: 129). Wahrgenommene kostenbezogene Risiken betreffen neben den Anschaffungskosten die Kosten für Ladeinfrastruktur. Als technisches Risiko wird die begrenzte elektrische Reichweite wahrgenommen und die dadurch potenziell geringere Flexibilität.

\section{Taxiunternehmen $(T)$}

Die allgemeine Einstellung zu E-Fahrzeugen ist überwiegend positiv. Ihre Nutzung in Taxiunternehmen wird deutlich negativer bewertet (vgl. Grausam/Parzinger/ Müller 2015: 165). Wahrgenommene Potenziale betreffen erhebliche Einsparungen durch geringere Stromkosten im Vergleich zu Benzinkosten sowie geringere Werkstattkosten durch einen niedrigen Verschleiß. Weitere Potenziale werden im Beitrag zum Umweltschutz gesehen und in einem höheren Komfort für Fahrgäste. Nutzungsmotive ergeben sich auch aus drohenden Fahrverboten, insbesondere für dieselbetriebene Fahrzeuge in städtischen Gebieten. Potenziale werden in der Teilnahme an einer Zukunftstechnologie gesehen sowie potenziellen Wettbewerbsvorteilen bei gesundheits- und umweltbewussten Kundinnen und Kunden.

Das wichtigste wahrgenommene Risiko ist die Notwendigkeit der Ablehnung von Kundinnen/Kunden aufgrund einer geringen elektrischen Reichweite. Die Befragten kritisieren die von den Herstellern angegebenen Reichweitenangaben, die unter realen Bedingungen unhaltbar wären. Selbst die während einer Fahrt angezeigten Reichweitenangaben werden unter realen Bedingungen als unzuverlässig empfunden. Bei Anlässen wie Silvester oder dem Ausfall öffentlicher Verkehrsmittel ist die Nachfrage und damit die Fahrleistung höher. Eine begrenzte elektrische Reichweite bedeutet hier finanzielle Verluste. Weitere Risiken betreffen organisatorische Abläufe. Im Zweischichtsystem ist die Wartezeit zwischen den Schichten zu kurz für ein langsames Laden (vgl. Harendt/Schumann/Wirth 2017: 107). Auch Zwischenladen wird negativ bewertet. Im Winter gilt der Einsatz von E-Fahrzeugen als problematisch. Niedrige Temperaturen, Heizung und die Nutzung von 


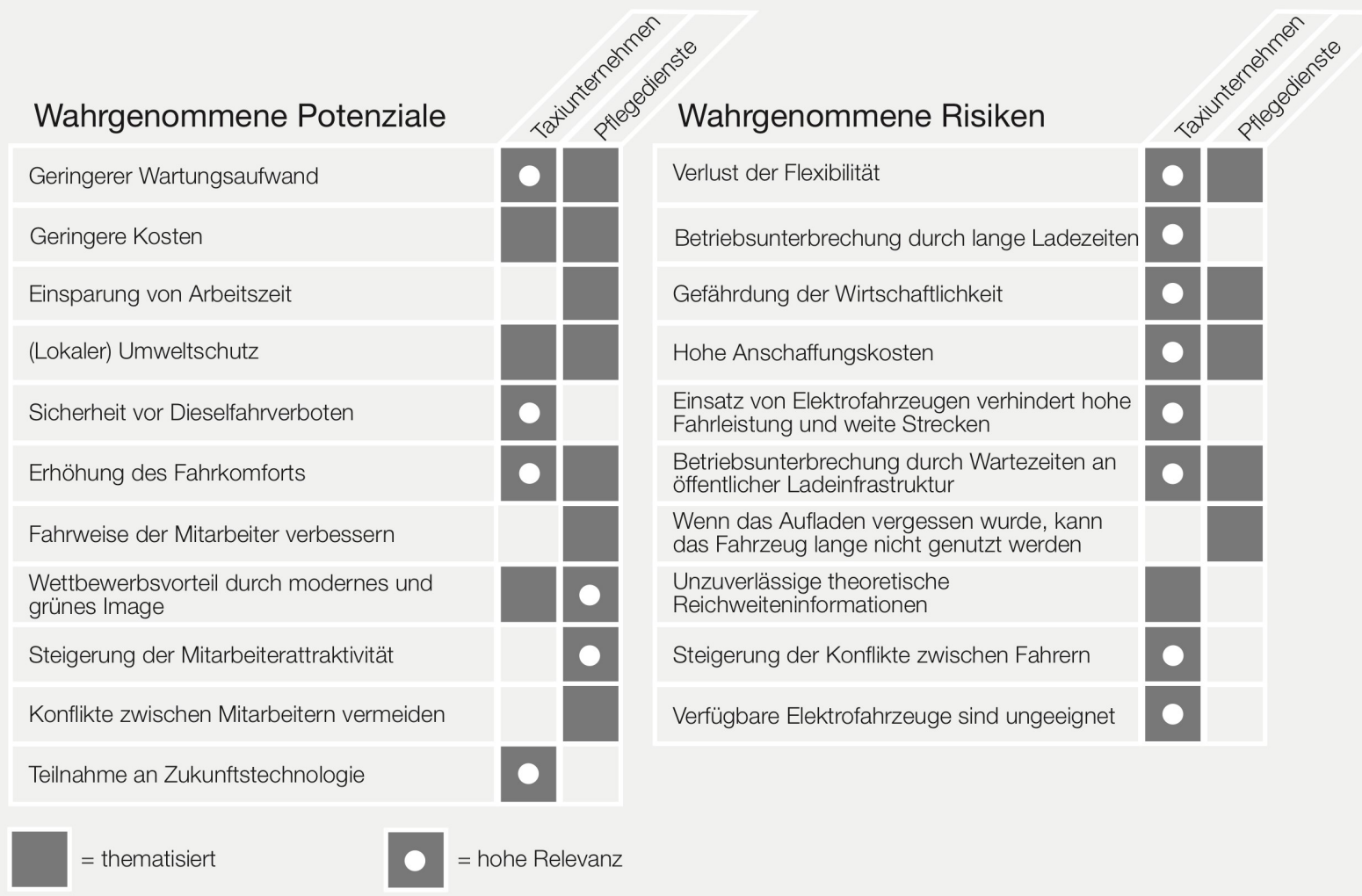

Abbildung 1: Wahrgenommene Potenziale und Risiken im Vergleich

Autoscheinwerfern belasten zusätzlich die Batterie und reduzieren gegebenenfalls die elektrische Reichweite (vgl. Goldschmidt/Richter/Pfeil 2019). E-Taxis nutzen Funktionen wie die Heizung nicht nur während der Kundenfahrt, sondern auch bei Wartezeiten am Taxistand.

Abbildung 1 stellt die durch die Stakeholdergruppen wahrgenommenen Potenziale und Risiken zusammengefasst dar. Der Vergleich ergibt starke Unterschiede, die insbesondere auf unterschiedlichen Arbeitsstrukturen und Arbeitsorganisation beruhen.

\subsection{Anforderungen an E-Fahrzeuge und Ladeinfrastruktur}

\subsubsection{Anforderungen an E-Fahrzeuge}

\section{Pflegedienste}

Vier der sechs Befragten in den Pflegediensten präferieren den Einsatz rein batterieelektrischer Fahrzeuge (vgl. Vogel 2016: 10). Plug-in-Hybride werden durch alle Befragten abgelehnt, sie würden dazu einladen, den konventionellen Motor zu verwenden, wenn die elektrische Reichweite nicht ausreicht. Ein Befragter hält $100 \mathrm{~km}$ für ausreichend, vier Befragte nennen 100-200 $\mathrm{km}$, ein anderer $200 \mathrm{~km}$. Der Letztgenannte hofft, dass E-Fahrzeuge dann nur einmal pro Woche aufgeladen werden müssen: „Es wäre schön, wenn wir die E-Fahrzeuge eine ganze Woche nicht laden müssten. Wir haben einen festen Tag in einer Woche, an dem die Wartung der Autos stattfindet. Das könnte man mit dem Laden der E-Fahrzeuge kombinieren" (Interview P3).

Die Mehrheit der Befragten hält schnellladefähige E-Fahrzeuge in ihrer Branche für nicht notwendig, sie seien zudem zu teuer (vgl. aber Uniper Technologies 2016: 107). Die Befragten wünschen leicht bedienbare und einfach zu wartende E-Fahrzeuge sowie ein flächendeckendes Wartungs- und Servicenetz für diese.

\section{Taxiunternehmen}

Die Reichweite eines E-Fahrzeugs ist eine wichtige Spezifikation zur Analyse der Bedürfnisse einer Interessengruppe (Ko/Kim/Nam et al. 2017). Zwei Befragte erwarten in ihrem professionellen Kontext eine Min- 


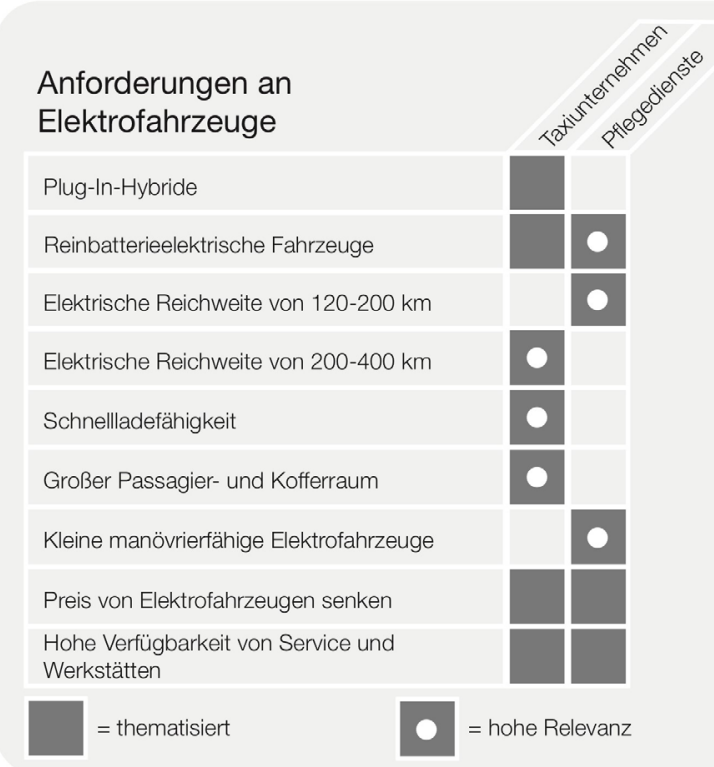

Abbildung 2: Anforderungen an E-Fahrzeuge im Vergleich

destreichweite von $190 \mathrm{~km}$. So können ganze Schichten wie auch Fahrten zu den Flughäfen ohne Zwischenladen durchgeführt werden. „Die überwiegende Mehrheit der Flughäfen, zu denen wir fahren, sind Düsseldorf und Köln. Ich muss in der Lage sein die Reise, inklusive der Rückfahrt nach Aachen, abzuschließen. Nun, eine $190 \mathrm{~km}$ lange Fahrt sollte möglich sein. Es sind 95 $\mathrm{km}$ pro Fahrt. Es ist mir wichtig, dass ich immer wieder zu meinen Ladestationen hier in Aachen zurückkehren kann“ (Interview T6).

Die Hälfte der Befragten benötigt eine Mindestreichweite von $400 \mathrm{~km}$. So könne ein durchschnittlicher Tag absolviert und weiter entfernte Ziele leicht erreicht werden. Nur ein Befragter wünscht eine Mindestreichweite von $1.000 \mathrm{~km}$, um im Falle von Zugausfällen im Grenzverkehr Fahrgäste zu weiter entfernten Zielen (Frankfurt, Brüssel, Paris) transportieren zu können. Auch wenn solche Fahrten selten sind, führt die Aussicht, derartige Kundenanfragen nicht bedienen zu können, zu einer negativen Wahrnehmung der elektrischen Reichweite. E-Fahrzeuge, die als Taxis genutzt werden, müssen zudem schnell ladbar sein (Asamer/ Reinthaler/Ruthmair et al. 2016: 234), z. B. zwischen Schichten. Weitere Bedarfe sind ein geringerer Kaufpreis (trotz geringerer Wartungskosten im Vergleich zu herkömmlichen Fahrzeugen) sowie eine größere E-Fahrzeug-Modellauswahl für ihre Zwecke. Da Taxis bis zu vier Fahrgäste mit Gepäck transportieren können müssen, muss das Fahrzeug groß sein. Werden diese
Anforderungen nicht erfüllt, kann das zu Schwierigkeiten beim Erwerb der Taxizulassung führen. Die verfügbaren Tesla-Modelle werden hinsichtlich Reichweite, Ladezeit und hochwertiger Ausstattung positiv bewertet, der Preis ist jedoch für kleine Unternehmen zu hoch. Zwei Befragte, die Plug-In-Hybride präferieren, argumentieren, dass der konventionelle Motor innen ein Gefühl der Sicherheit gibt, auch lange Kundenreisen antreten zu können. Ein anderes Argument ist, dass die Taxibranche zunehmend Parallelhybride einsetze und Plug-InHybride als E-Fahrzeug-Variante bevorzuge. Langfristig präferiert die Mehrheit der Befragten jedoch den Einsatz rein elektrischer Fahrzeuge (vgl. Hagmann/Langbroek 2019).

Abbildung 2 zeigt die Befunde im Vergleich. Die Unterschiede betreffen insbesondere Fahrzeugeigenschaften wie Reichweite, Größe, Schnellladen und Manövrierbarkeit.

\subsubsection{Anforderungen an die Ladeinfrastruktur}

\section{Pflegedienste}

Alle Befragten präferieren aus der Sicht ihres Berufsalltags das Laden von E-Fahrzeugen über Nacht (vgl. Grausam/Parzinger/Müller 2015; Ehrler/Luft/Elektirikçi et al. 2016), alternativ zwischen Früh- und Spätschicht. Die Dauer eines Ladevorgangs hängt von E-Fahrzeug und Ladeinfrastruktur ab. Der Mehrheit der Befragten reicht langsames Laden (ähnlich Uniper Technologies 2016: 42). Die Option des Schnellladens auf dem Betriebsgelände würde jedoch Pflegefahrzeugen (vgl. Ehrler/Luft/Elektirikçi et al. 2016), Dienstfahrzeugen für andere Zwecke wie auch Mitarbeiterfahrzeugen, die dort stehen, mehr Flexibilität und Sicherheit bringen. „Für den schlimmsten Fall möchte ich es so ausdrücken: wenn jemand den Tag oder die Schicht vorher wirklich nicht geladen hat. So etwas kann passieren. Dann ist es wichtig, dass Schnellladen möglich ist und dass Sie es nutzen können, wenn auch nur für diese Situationen" (Interview P5).

Das Laden sollte in erster Linie auf dem Betriebsgelände erfolgen. Ein Pflegedienst erlaubt dem Personal, Flottenfahrzeuge mit nach Hause zu nehmen (vgl. Grausam/Parzinger/Müller 2015). Dann müssen bei E-Fahrzeugen daheim Lademöglichkeiten installiert sein. Der Pflegedienst würde nach eigener Aussage alle Stromkosten übernehmen und auch die private Nutzung unterstützen. Ist eine Installation zum Aufladen nicht möglich, muss die öffentliche Ladeinfrastruktur in der Nähe des Wohnsitzes genutzt werden. 


\section{Taxiunternehmen}

Der Einsatz von E-Fahrzeugen als Taxi ist an eine flächendeckend verfügbare Ladeinfrastruktur gebunden. Sie sollte diverse Ladearten unterstützen (vgl. Helmus/ van den Hoed 2015: 595), eine hohe Anzahl von Ladepunkten bieten und zuverlässig funktionieren. Technische Störungen wie auch Umstände, die den Ladevorgang behindern (z. B. Falschparker/-innen) müssen minimiert werden. Die Installations-, Betriebs- und Wartungskosten für Ladeinfrastruktur sollten sinken wie auch die Ladezeit für den flexiblen Einsatz von E-Fahrzeugen (vgl. Teuschl/Deiml/Ackerl 2017: 34).

Spezielle Anforderungen betreffen den Standort der Ladestationen. Taxiunternehmen wollen primär am Taxistand laden (vgl. Grausam/Parzinger/Müller 2015: 162; Goldschmidt/Richter/Pfeil 2019: 14) und zwar innerhalb von 20 Minuten (Schnellladung). Vorbehalte und Planungsbedarf betreffen das Design der Taxistände. Die Befragten thematisieren potenzielle Konflikte zwischen konkurrierenden Taxifahrern/-innen bzw. Taxiunternehmern/-innen in Bezug auf die Nutzungsdauer und die Häufigkeit der Nutzung der Ladeinfrastruktur. „Sie sind alle Wettbewerber auf dem gleichen Markt. Sie sind natürlich Gegner. Und dann wird ein Unternehmen sagen: Großartig, sie nutzen es länger als wir, mal wieder, weil sie größer sind oder so. Solche Probleme bekommt man leicht" (Interview T6).

Akzeptanzrelevant ist die Positionierung der Ladepunkte am Taxistand. Da die Fahrgäste in das erste Fahrzeug der Warteschlange steigen, fürchten die Taxifahrer/-innen, bei kabelgebundenem Ladevorgang nicht nachrücken zu können. Die Platzierung der Ladestation(en) am hinteren Ende des Taxistandes erzeugt Kundenverlust, die Platzierung an der Spitze Konflikte mit anderen Taxifahrern: „Wenn die Hälfte der wartenden Taxis [am Hauptbahnhof] elektrisch sind, benötigen sie zehn Ladestationen. Wo willst du sie hinstellen? Legst du sie vorn oder hinten an? Wenn sie hinten sind, wählen Kunden Taxis, die die ersten in der Reihe sind. Wenn du sie vor die anderen stellst, werden sie [Taxifahrer mit konventionellen Fahrzeugen] anfangen, sich zu beschweren" (Interview T2).

Konflikte entstehen, wenn in der Schlange stehende herkömmliche Taxis die Ladesäulen blockieren. Als Lösungsansätze werden Halteverbotszonen und Taxistandplätze ausschließlich für E-Taxis genannt.

Verfügt das Taxiunternehmen über ein Betriebsgelände, sollte dort Ladeinfrastruktur installiert werden (vgl. Grausam/Parzinger/Müller 2015: 162). Dies planen zwei befragte Unternehmen. Eins dieser größeren Taxiunternehmen erlaubt seinen Fahrerinnen und Fahrern, gelegentlich Fahrzeuge mit nach Hause zu nehmen. Bei E-Fahrzeugen soll die Mitarbeiterin/der Mitarbeiter die öffentliche Ladeinfrastruktur in der Nähe der eigenen Wohnung nutzen. Die Installation einer eigenen Ladestation lohnt nicht, weil die Fahrer/-innen oft bei verschiedenen Taxiunternehmen arbeiten und/oder nach kurzer Zeit den Arbeitgeber wechseln. Insgesamt ist die Nutzungsbereitschaft (halb-)öffentlicher Ladeinfrastruktur geringer als in anderen Studien (vgl. aber Grausam/ Parzinger/Müller 2015: 162).

Die Befragten beschränken Schnellladen auf die Ladeinfrastruktur an Taxiständen oder im Zweischichtsystem (vgl. aber Helmus/van den Hoed 2015). Im Zweischichtsystem sind die Wartezeiten zwischen den Schichten zu kurz, um das E-Taxi bei langsamer Ladegeschwindigkeit vollständig zu laden. Wird das E-Taxi nur in einer Schicht pro Tag genutzt, reiche langsames Laden - bevorzugt an dem Ort, an dem das Taxi geparkt wird, nach der Schicht am Abend oder über Nacht. „Ein Fahrzeug steht hier immer am Abend bei mir zu Hause. Das Fahrzeug kann über Nacht aufgeladen werden. Am Morgen kommt der neue Fahrer vorbei und kann das voll aufgeladene Fahrzeug für seine Schicht nutzen. Dann kommt das andere Fahrzeug zurück" (Interview T3).

E-Fahrzeuge mit begrenzter Reichweite müssen während der Schicht geladen werden, bei langen Fahrten nach der Rückkehr. Ladezeit und Ladedauer können je nach gefahrener Strecke und Restreichweite stark variieren. Das Zwischenladen während einer Schicht bewerten alle Befragten eher negativ, insbesondere wenn das Taxi zu einem Ort außerhalb des normalen Einsatzgebietes gebracht werden muss. Bevorzugte Orte des Zwischenladens sind der Taxistand sowie Orte, an denen die offiziell geforderten Pausen eingelegt werden. „Wenn das Schnellladen wirklich so schnell wäre, könnte man während der Schicht laden. Du könntest während der offiziellen Pause [...] laden. Dann tue ich wenigstens etwas Nützliches in der Pause, die ich nehmen muss" (Interview T6).

Die Zeit, in der das Fahrzeug ungenutzt bleibt, sollte möglichst gering sein. Im Zweischichtsystem mit Tag- und Nachtwechsel sind die längsten ungenutzten Zeiten die frühen Morgenstunden. In dieser Zeit sollte der Hauptladevorgang stattfinden.

Die Befragten thematisieren selbstinitiiert alternative E-Fahrzeugkonzepte. Ein Befragter ist an der Nutzung von Wasserstofffahrzeugen interessiert, scheut jedoch die Kosten und vermisst die dazu nötige Infrastruktur. Es werden auch alternative Ladekonzepte angesprochen. Die kabelgebundene Ladung wird von zwei Befragten als zu langsam und umständlich bewertet. Sie erfülle 
nicht die besonderen Mobilitätsbedürfnisse der Taxiunternehmen. Batteriewechselstationen seien eine Möglichkeit, den Akku in kurzer Zeit zu laden. Zwei Befragte favorisieren die induktive Ladung. So sei das Laden am Taxistand weniger problematisch, da die Fahrzeuge nicht mit einer Station verbunden sind.

Vergleicht man die Befunde auf Stakeholdergruppen bezogen (vgl. Abbildung 3), zeigen sich wieder deutliche Unterschiede, z. B. in puncto Ladeort oder Ladegeschwindigkeit.

\subsection{Informations- und Partizipationsbedarf}

\section{Pflegedienste}

Für eine fundierte Entscheidung für oder gegen den Einsatz von Elektromobilität wünschen sich die befragten Entscheiderinnen und Entscheider Erfahrungsberichte anderer Pflegedienste, die erfolgreich E-Fahrzeuge in ihre Flotte integriert haben, etwa um Nutzungsrisiken besser einschätzen zu können. Drei Befragte bezweifeln die in der Berichterstattung überwiegend positiv dargestellte Ökobilanz von E-Fahrzeugen (von der Herstellung bis zum Batterie-Recycling) im Vergleich zu konventionellen Fahrzeugen. Sie wünschen zudem eine übersichtliche Zusammenstellung von Vor- und Nachteilen (z. B. als on- oder offline verfügbaren Flyer).

Besonders wichtig für die Entscheidung, die eigene Flotte auf E-Fahrzeuge umzustellen, sind Förderoptionen. Zwei Befragte wünschen detaillierte Information zu Fördermöglichkeiten - auf einer Internetseite, in einem Flyer oder als persönliche Beratung durch Automobilhersteller oder -händler. Sie wollen zentral über Förderprojekte informiert werden. Bei Projekten, die gewerbliche E-Mobilität fördern, sollte sich die Projektleitung proaktiv an (regionale) Pflegedienste wenden und diese aktiv über das Vorhaben informieren sowie Kontakt halten. Dies erlaube den Pflegediensten, Projektziele frühzeitig strategisch einzubeziehen. Im Projektverlauf verlagert sich das Interesse auf Projektfortschritte. Bei Ladeinfrastrukturausbauprojekten wollen sie über Meilensteine, potenzielle Verzögerungen in der Umsetzung sowie die entstehende Ladepunkt-Landschaft informiert werden. Das ,Wie' des Informierens wird kontrovers gesehen. Einige präferieren aktuelle Information per Website, andere lehnen dies als veraltet $a b$ und präferieren Social-Media-Beiträge. Auch E-Mail-Newsletter werden kontrovers gesehen; es gäbe zu viele, das Format wird schnell in der Menge übersehen.
Anforderungen an

Ladeinfrastruktur

Laden auf Betriebsgelände über Nacht

Laden an Wohnsitz des Unternehmers über

Nacht

Laden an Wohnsitz der Mitarbeiter über Nacht

Laden an halböffentlicher Infrastruktur (Restaurant/ Supermarkt) in Pausen

Laden an halböffentlicher Infrastruktur

(Parkhaus), für Mitarbeiter gemietet über Nacht

Laden an öffentlicher Infrastruktur nahe des

Wohnortes der Mitarbeiter über Nacht

Laden im öffentlichen Raum an einer

Ladestation, die nur für Flotten verfügbar ist

Mitarbeiter/innen-Elektrofahrzeuge auf

Betriebsgelände laden

Ladekarten für öffentliche Ladeinfrastruktur

Schnellladeinfrastruktur für Notfall

Hohe Funktionalität und Zuverlässigkeit

Erhöhung der Ladegeschwindigkeit

$=$ thematisiert

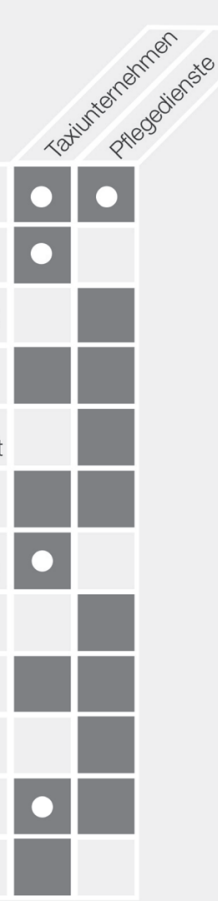

(2)

thematisiert

= hohe Relevanz

Abbildung 3: Anforderungen an die Ladeinfrastruktur im Vergleich

Zwei Befragte wollen Trainings, die die Mitarbeiter/ -innen auf die tägliche Arbeit mit E-Fahrzeugen vorbereiten und die Umstellung von konventionellen zu E-Fahrzeugen effektiv unterstützen. Potenzielle Schulungsthemen sind energiesparendes, geräuschloses Fahren sowie Laden. Für die Trainings werden verständlich aufbereitete Trainingsunterlagen, gegebenenfalls von Fahrzeugherstellern, gewünscht.

\section{Taxiunternehmen}

Trotz Interesse an Elektromobilität sind aktuelle technische Entwicklungen eher unbekannt. Vier Befragte gehen - wie die Pflegedienste - von einem mehrstündigen Ladevorgang aus. Die Hälfte der Befragten kennt die Option des Schnellladens nicht. Vereinzelt wird angenommen, dass Schnellladen der Fahrzeugbatterie schadet und die Reichweite des E-Fahrzeugs reduziert. Fast alle Befragten ( 5 von 6 ) berichten, dass zum Zeitpunkt der Befragung keine Modelle existieren, die ihre Mobilitätsanforderungen (Reichweite, Ladezeit, Passagierkapazität) erfüllen und durch Kleinunternehmen finanzierbar sind. Sie sind unsicher, welche E-Fahrzeuge als Taxi ausgestattet und zugelassen werden können. 
Insgesamt besteht erheblicher Bedarf nach branchenspezifisch aufbereiteten Informationen zu E-Fahrzeugen. Die Mehrzahl der Befragten ist an Informationsbroschüren interessiert, die die Vorteile der Nutzung von E-Fahrzeugen bezogen auf Nutzungsszenarien (z. B. Einsatz im Stadtverkehr) im Vergleich zu konventionellen Fahrzeugen darstellen. Relevante Themen sind (Strom-)Verbrauch, Versicherungskosten, Wartungskosten, technische Spezifika, wie die Energierückgewinnung (Rekuperation), sowie erwarteter Wertverlust der E-Fahrzeuge. Da gewerblich genutzte Fahrzeuge nach kurzer Nutzungsdauer weiterverkauft werden, ist das Interesse an Informationen zur Wertstabilität von E-Fahrzeugen hoch. Wiederholt wird das Thema Stromkosten angesprochen. Zwei Befragte wollen eine exemplarische Aufstellung voraussichtlicher Stromkosten, da innen derzeit Erfahrungswerte fehlen, was die Gewinnabschätzung erschwert. Die Befragten gehen davon aus, dass die Kosten für Strom geringer sind als für konventionelle Kraftstoffe; sie benötigen als Unternehmer jedoch genauere Angaben. Unklar ist weiter, inwiefern sich die Versicherungskosten bei einer Umstellung auf E-Fahrzeuge ändern (sie sind für Taxis deutlich höher als für privat genutzte Fahrzeuge). Die Broschüre sollte durch die Industrie- und Handelskammer angeboten und distribuiert werden, da sie zentrale Ansprechpartnerin und Interessenvertreterin der Taxiunternehmen sind.

Zwei Befragte wünschen Informationen zu Fördermöglichkeiten - in einer übersichtlichen Darstellung oder in einem Beratungsgespräch - zu der Höhe der Förderung, eventuelle Einschränkungen und Voraussetzungen sowie dem Beantragungsvorgang. Weitere Themen sind steuerliche Vorteile und Vergünstigungen bei der Anschaffung und Nutzung von E-Fahrzeugen und Ladeinfrastruktur.

Von den Taxiunternehmer/-innen kennt niemand das zugrunde liegende Ladeinfrastrukturausbauprojekt. Sie wollen früh (bei Projektbeginn) über derartige Projekte informiert werden, um zu prüfen, ob und wie sie diese für die Umstellung auf Elektromobilität nutzen können. Information wird als Bringschuld des Projekts gesehen. Bei Projekten zum Ausbau der regionalen Ladeinfrastruktur ist allen Befragten wichtig, zeitnah und kontinuierlich über Fortschritte, Verzögerungen und Probleme des Ausbaus informiert zu werden, um sich strategisch darauf einstellen zu können. Zwei Befragte interessiert besonders die Errichtung neuer, nutzbarer Ladepunkte. Für Informationszwecke werden zwei Formate präferiert: E-Mail-Newsletter und Informationsveranstaltungen für Unternehmer/-innen, die sich für den Wechsel zu E-Fahrzeugen interessieren. In der Informationsver- anstaltung werden Möglichkeiten der Umstellung dargestellt, die Unternehmen können Fragen stellen und sich beraten lassen. Bei konkretem Interesse sehen sie die Notwendigkeit einer persönlichen Konsultation durch E-Mobilitäts-Experten. Zwei Taxiunternehmer nennen exemplarisch den durch die Aachener Stadtwerke betriebenen E-Store als gute Quelle für fundierte persönliche Konsultation. Dort können sich Interessierte zu E-Mobilität, Lademöglichkeiten und Fördermitteln beraten lassen sowie E-Fahrzeuge Probe fahren.

Im Falle der geplanten Installation von Schnellladeinfrastruktur an Taxiständen sollten vorab alle Taxiunternehmen darüber informiert werden, damit sie frühzeitig potenzielle Vor- und Nachteile strategisch abschätzen bzw. sich aktiv in die Planung einbringen können.

\section{Diskussion und Fazit}

Die Stichprobe dieser Studie ist mit zwölf Teilnehmerinnen und Teilnehmern sehr klein. Restriktionen bei der Suche von Teilnehmerinnen und Teilnehmer ergaben sich aus dem Erhebungsfokus (lokale Unternehmen, die zu E-Mobilität wechseln wollen) und der Spezifik der Taxibranche. Durch die geringe Stichprobengröße lassen sich Erkenntnisse nur bedingt generalisieren, sie müssen in größeren bzw. in weiteren Studien geprüft werden. Angesichts technischer Fortschritte und des fortlaufenden Prozesses der Energiewende sollte regelmäßig geprüft werden, wie sich Anforderungen und Wahrnehmungen verändern, um Förderung, Informationsmaßnahmen und Partizipationsangebote darauf abstimmen zu können. Wichtig ist die Berücksichtigung lokaler Rahmenbedingungen. Hier bieten sich kleinere, explorative Studien an.

Die in der Studie Befragten haben eine lange Berufserfahrung in ihrer Branche und umfangreiches Wissen zu Abläufen in dieser. Sie sind am Wechsel zu Elektromobilität interessiert und für Entscheidungen zuständig. Der Entscheidungsprozess unterliegt branchenspezifischen Erwägungen und ist lokal geprägt (z. B. durch die Lage im Dreiländereck). Es zeigen sich starke Branchenunterschiede in Bezug auf Anforderungen an E-Fahrzeuge, ihre gewerbliche Nutzung in Flotten, das Design der Ladeinfrastruktur sowie Informations- und Partizipationsbedarfe. Die Unterschiede ergeben sich aus der Arbeitsorganisation in den Unternehmen und dem Nutzungszweck. Die Bereitschaft zur aktiven Umstellung auf Elektromobilität ist vorhanden, jedoch unterschiedlich stark. Am stärksten ist das Interesse bei den Pflegediensten. Das Konzept passt gut zu ihrer Arbeitsor- 
ganisation und ihren Rahmenbedingungen (z. B. kurze, gut planbare Wege). Die Gruppe der Taxiunternehmen ist dagegen vergleichsweise zurückhaltend. Dies lässt sich unter anderem durch eine hohe Laufleistung, nicht planbare Routen und den direkten Zusammenhang von Umsatz und Fahrzeugauslastung erklären. Relevant für die Kaufentscheidung sind unter anderem die hohen Anschaffungskosten für Fahrzeuge und Ladeinfrastruktur. Insbesondere für kleine Taxiunternehmen oder selbstfahrende Taxiunternehmer/-innen sind die Initialkosten eine große Hürde. Akzeptanzrelevant für Taxiunternehmen ist auch das Laden an Taxiständen und ein Design dieser Orte, das ihre Bedürfnisse erfüllt. Beide betrachteten Zielgruppen gestatten Mitarbeiterinnen und Mitarbeitern partiell, Dienstfahrzeuge mit nach Hause zu nehmen. Wenn sie E-Fahrzeuge nutzen, benötigen sie Ladeinfrastruktur am Wohnsitz oder öffentlich verfügbar in der Nähe. Der Ausbau der Ladeinfrastruktur sollte nicht zuletzt deshalb integrativ erfolgen.

Der gezielte lokale Ausbau von Ladeinfrastruktur bedingt, dass sich die Verantwortlichen der Unterschiedlichkeit zielgruppenbezogener Bedarfe und Erwartungen bewusst sind. Stakeholderprofilkarten ermöglichen, Unterschiede schnell erfassbar darzustellen. Sie verdichten Einzelinformationen, gewichten Themen, sensibilisieren für Unterschiede und indizieren relevante Themen für Informationsmaßnahmen. Bei beiden Zielgruppen ist der Wissensstand zu E-Fahrzeugen gering und teilweise veraltet. Taxiunternehmen haben im Vergleich zu Pflegediensten einen deutlich höheren Informationsbedarf zu technischen Aspekten von Elektrofahrzeugen sowie der ökonomischen Betrachtung ihrer Nutzung. Sie benötigen branchenspezifisch aufbereitetes Wissen in Form von Broschüren sowie die Option der Beratung. Das Interesse der Pflegedienste richtet sich primär auf die Sicherung einer störungsfreien Versorgung ihrer Patientinnen und Patienten. Sie würden bei der Umstellung auf Elektromobilität von Erfahrungsberichten anderer Pflegedienste profitieren, die bereits erfolgreich E-Fahrzeuge in ihre Flotte integriert haben.

Beide Zielgruppen sind an Fördermitteln interessiert und wollen effizient zu Förderoptionen und Förderprojekten in ihrem unmittelbaren Umfeld informiert werden - an zentraler Stelle oder individuell. Es gibt bereits im Internet Übersichten zu Projekten, zum Beispiel auf den Seiten von Förderern, diese reichen jedoch offensichtlich nicht aus, um die Informationsbedarfe zu decken bzw. werden von den Zielgruppen nicht wahrgenommen. Hier zeichnen sich Handlungsbedarfe der für Projekte Verantwortlichen im Sinne einer besser wahrnehmbaren Öffentlichkeitsarbeit ab. Als potenzielle Distributoren bzw. Vermittler wurde unter anderem die Industrie- und Handelskammer genannt. Pflegedienste wollen frühzeitig proaktiv durch die Projektleitung kontaktiert und informiert werden, um Chancen für ihr Unternehmen prüfen zu können. Taxiunternehmen wollen partizipieren können, wenn es um das Design und die Ausrüstung von Taxiständen mit Ladeinfrastruktur geht. Hier bieten sich Formate wie Gestaltungsworkshops an. Als geeignete Mittel des Informierens über den Stand des Infrastrukturausbaus werden Website, Social Media (Pflegedienste) und E-Mail-Newsletter (Taxiunternehmen) genannt. Insgesamt zeichnet sich ab, dass die kommunikative Begleitung professioneller Nutzer/-innen die Branchenspezifik von Sichten, Themen und Bedarfen aufgreifen und ernst nehmen muss. Gut aufbereitete Informationen, zu den Themen, die die Zielgruppe bewegen, können wesentlich dazu beitragen, Befürchtungen und Unsicherheiten in Bezug auf den Vollzug von Arbeit und den unternehmerischen Erfolg abzubauen. Angesichts der Schnelligkeit technologischer Entwicklungen sind derartige Angebote aber nur dann sinnvoll, wenn kleine und mittelständische Unternehmen Aspekte der Elektromobilität im Kontext ihrer Branche fundiert abwägen können. Die Möglichkeit des Zugriffs auf gut aufbereitete Informationsangebote, die auf die Bedarfe der Branche reagieren, spielt daher eine wichtige Rolle. Um Konflikte in der Infrastrukturnutzung zu vermeiden, sollte die Perspektive zukünftiger Nutzung in die Gestaltung des Ladeinfrastrukturausbaus einfließen und Zielgruppen früh partizipativ einbezogen werden.

Förderhinweis: Das diesem Beitrag zugrunde liegende Vorhaben wurde mit Mitteln des Bundesministeriums für Wirtschaft und Energie unter dem Förderkennzeichen 01MZ18006G gefördert (Projekt ALigN - Ausbau der Ladeinfrastruktur durch gezielte Netzunterstützung. Teilvorhaben: Erforschung von technischen, ökonomischen und kommunikativen Konzepten zum nachhaltigen Ausbau von Ladeinfrastruktur). Die Verantwortung für den Inhalt dieser Veröffentlichung liegt bei den Autorinnen.

\section{Literatur}

Asamer, J.; Reinthaler, M.; Ruthmair, M.; Straub, M.; Puchinger, J. (2016): Optimizing Charging Station Locations for Urban Taxi Providers. In: Transportation Research Part A: Policy and Practice 85, 3, 233-246. doi: 10.1016/j.tra.2016.01.014

Becker, S.; Renn, O. (2019): Akzeptanzbedingungen politischer Maßnahmen für die Verkehrswende: Das Fallbeispiel Berliner 
Mobilitätsgesetz. In: Fraune, C.; Knodt, M.; Gölz, S.; Langer, K. (Hrsg.): Akzeptanz und politische Partizipation in der Energietransformation. Wiesbaden, 109-130. doi: 10.1007/9783-658-24760-7_6

Bozem, K.; Nagl, A.; Rath, V.; Haubrock, A. (2013): Elektromobilität: Kundensicht, Strategien, Geschäftsmodelle. Wiesbaden. doi: 10.1007/978-3-658-02628-8.

Dumortier, J.; Siddiki, S.; Carley, S.; Cisney, J.; Krause, R. M.; Lane, B. W.; Graham, J. (2015): Effects of Providing Total Cost of Ownership Information on Consumers' Intent to Purchase a Hybrid or Plug-In Electric Vehicle. In: Transportation Research Part A: Policy and Practice 72, 2, 71-86. doi: 10.1016/j. tra.2014.12.005

Ehrler, V.; Luft, D.; Elektirikçi, S.; Schöder, D.; Walter, D.; Sternkopf, B. (2016): Projekt Smart E-User - Konzept für elektrische Stadtlogistik. Im Schaufenster Elektromobilität der Region Berlin/Brandenburg. Abschlussbericht AP1. Nutzerprofile und Szenarienbildung. Berlin.

Fröschle, N. (2017): Engineering von neuen Bürgerbeteiligungsinstrumenten am Beispiel von Elektromobilität, Feinstaub und Luftreinhaltung. In: HMD Praxis der Wirtschaftsinformatik 54, 4, 502-517. doi: 10.1365/ s40702-017-0325-2

Globisch, J.; Dütschke, E.; Wietschel, M. (2018): Adoption of electric vehicles in commercial fleets: Why do carpool managers campaign for BEV procurement? In: Transportation Research Part D: Transport and Environment 64, 11, 122-133. doi: 10.1016/j.trd.2017.10.010

Gnann, T.; Plötz, P.; Funke, S.; Wietschel, M. (2015): What is the Market Potential of Plug-In Electric Vehicles as Commercial Passenger Cars? A Case Study from Germany. In: Transportation Research Part D: Transport and Environment 37, 6, 171-187. doi: 10.1016/j.trd.2015.04.015

Gölz, S.; Langer, K.; Becker, A.; Götte, S.; Marxen, T.; Berneiser, J. (2019): Akzeptanz und Konflikte als Zustände regionaler sozialer Prozesse. Anwendung eines transdisziplinären Analyserahmens. In: Fraune, C.; Knodt, M.; Gölz, S.; Langer, K. (Hrsg.): Akzeptanz und politische Partizipation in der Energietransformation, 85-105. Wiesbaden. doi: 10.1007/9783-658-24760-7 5

Goldschmidt, R.; Richter, A.; Pfeil, R. (2019): Active Stakeholder Involvement and Organisational Tasks as Factors for an Effective Communication and Governance Strategy in the Promotion of E-Taxis. Results from a Field Research Lab. In: Energy Policy 135, 110848, 1-17. doi: 10.1016/j. enpol.2019.06.056

Grausam, M.; Parzinger, G.; Müller, U. (2015): Elektromobilität in Flotten. Handlungsleitfaden. Handlungsempfehlungen zur Integration von Elektromobilität in Flotten für Fuhrparkbetreiber. Berlin.

Hagman, J.; Langbroek, J. H. M. (2019): Conditions for Electric Vehicle Taxi: A Case Study in the Greater Stockholm Region. In: International Journal of Sustainable Transportation 13, 6, 450-459. doi: 10.1080/15568318.2018.1481547

Harendt, B.; Schumann, D.; Wirth, M. (2017): SchaufensterProgramm Elektromobilität. Abschlussbericht der Begleit- und Wirkungsforschung 2017. Frankfurt am Main.

Hellmuth, N.; Jakobs, E.-M. (2019): Partizipationsformate für Stromnetzausbauprojekte im ruralen Raum. In: Fraune, C.; Knodt, M.; Gölz, S.; Langer, K. (Hrsg.): Akzeptanz und politische Partizipation in der Energietransformation. Wiesbaden, 183-207. doi: 10.1007/978-3-658-24760-7_9

Helmus, J.; van den Hoed, R. (2015): Unraveling User Type Characteristics: Towards a Taxonomy for Charging Infrastructure. In: World Electric Vehicle Journal 7, 4, 589-604. doi: 10.3390/wevj7040589

Jakobs, E.-M. (2019): Technikakzeptanz und -kommunikation Ein vielschichtiges Konstrukt. In: Fraune, C.; Knodt, M.; Gölz, S.; Langer, K. (Hrsg.): Akzeptanz und politische Partizipation in der Energietransformation. Wiesbaden, 301-321. doi: 10.1007/978-3-658-24760-7_14

Kampker, A.; Deutskens, C.; Maue, A.; Hollah, A. (2016): Elektromobile Logistik. In: Deckert, C. (Hrsg.): CSR und Logistik. Spannungsfelder Green Logistics und City-Logistik. Berlin, 293-308. doi: 10.1007/978-3-662-46934-7_15

Kaplan, S.; Gruber, J.; Reinthaler, M.; Klauenberg, J. (2016): Intentions to Introduce Electric Vehicles in the Commercial Sector: A Model based on the Theory of Planned Behaviour. In: Research in Transportation Economics 55, 6, 12-19. doi: 10.1016/j.retrec.2016.04.006

Ketelaer, T.; Kaschub, T.; Jochem, P.; Fichtner, W. (2014): The Potential of Carbon Dioxide Emission Reductions in German Commercial Transport by Electric Vehicles. In: International Journal of Environmental Science and Technology 8, 11, 21692184. doi: 10.1007/s13762-014-0631-y

Kis, A.; Augustin, M.; Augustin, J. (2017): Regional Healthcare Delivery and Demographic Change in Germany - Scenarios for Dermatological Care in 2035. In: Journal der Deutschen Dermatologischen Gesellschaft 15, 12, 1199-1209. doi: 10.1111/ ddg.13379

Ko, J.; Kim, D.; Nam, D.; Lee, T. (2017): Determining Locations of Charging Stations for Electric Taxis Using Taxi Operation Data. In: Transportation Planning and Technology 40, 4, 420-433. doi: 10.1080/03081060.2017.1300243

Kraftfahrt-Bundesamt (2020): Pressemitteilung Nr. 01/2020 Fahrzeugzulassungen im Dezember 2019 - Jahresbilanz - korrigierte Fassung. Flensburg. https://www.kba.de/DE/ Presse/Pressemitteilungen/2020/Fahrzeugzulassungen/ pm01_2020_n_12_19_pm_komplett.html (25.08.2020).

Krause, R. M.; Carley, S. R.; Bradley, W. L.; Graham, J.D. (2013): Perception and Reality: Public Knowledge of Plug-In Electric Vehicles in 21 U.S. Cities. In: Energy Policy 63, 12, 433-440. doi: 10.1016/j.enpol.2013.09.018

Krips, D. (2017): Stakeholdermanagement. Kurzanleitung Heft 5. Berlin. doi: 10.1007/978-3-662-55634-4

Mayring, P. (2015): Qualitative Inhaltsanalyse. Grundlagen und Techniken. Weinheim.

Nanz, P.; Fritsche, M. (2012): Handbuch Bürgerbeteiligung. Verfahren und Akteure, Chancen und Grenzen. Bonn.

NOW - Nationale Organisation Wasserstoff- und Brennstoffzellentechnologie (2018): Urbaner Wirtschaftsverkehr. Berlin.

Plötz, P.; Gnann, T.; Ullrich, S.; Haendel, M.; Globisch, J.; Dütschke, E.; Wietschel, M.; Held, M. (2014): Elektromobilität in gewerblichen Flotten. Karlsruhe.

Reimer, E.; Jakobs, E.-M.; Borg, A.; Trevisan, B. (2015): New ways to develop professional communication concepts. In: Proceedings of the IEEE International Professional Communication Conference 97-103. doi: 10.1109/IPCC.2015.7235788 
Renn, O. (2015): Akzeptanz und Energiewende. Bürgerbeteiligung als Voraussetzung für gelingende Transformationsprozesse. In: Heimbach-Steins, M. (Hrsg.): Ethische Herausforderungen der Energiewende. Münster, 133-154. = Jahrbuch Christliche Sozialwissenschaften 56.

Sellmair, R.; Hamacher, T. (2014): Optimization of Charging Infrastructure for Electric Taxis. In: Transportation Research Record. Journal of the Transportation Research Board 2416, 1 , 82-91. doi: $10.3141 / 2416-10$

Teuschl, G.; Deiml, M.; Ackerl, M. (2017): Laden - eine neue Herausforderung für das Nutzfahrzeug. In: ATZelektronik 12, 1, 32-37. doi: 10.1007/s35658-017-0084-4

Tian, Z.; Jung, T.; Wang, Y.; Zhang, F.; Tu, L.; Xu, C.; Tian, C.; Li, X.-Y. (2016): Real-Time Charging Station Recommendation System for Electric-Vehicle Taxis. In: Transactions on Intelligent Transportation Systems 17, 11, 3098-3109. doi: 10.1109/TITS.2016.2539201

Uniper Technologies (2016): Gewerblich operierende ElektroKleinflotten (GO-ELK). Abschlussbericht. Gelsenkirchen.

Vogel, M. (2016): Elektromobilität in gewerblichen Anwendungen. Online-Befragung unter Expertinnen und Experten, (zukünftigen) Anwenderinnen und Anwendern sowie Dienstleistungsunternehmen im Kontext gewerblich zugelassener E-Fahrzeuge in allen Branchen. Berlin.

Wang, G.; Zhang, Y.; Fang, Z.; Wang, S.; Zhang, D. (2020): FairCharge: A Data-Driven Fairness-Aware Charging Recommendation System for Large-Scale Electric Taxi Fleets. In: Proceedings of the ACM on Interactive, Mobile, Wearable and Ubiquitous Technologies 4, 1, 28, 1-28. doi: $10.1145 / 3381003$

Witte, C.; Klumpp, M.; Keuschen, T.; Zelewski, S. (2013): AHPAnalyse der Prozessanpassungen in der Logistik beim Einsatz von Elektronutzfahrzeugen. In: Schenk, M.; Zadek, H.; Müller, G.; Richter, K.; Seidel, H. (Hrsg.): 18. Magdeburger Logistiktage $\square$ Sichere und nachhaltige Logistik. Magdeburg, 43-51.

Zhang, D.; Sun, L.; Li, B.; Chen, C.; Pan, G.; Li, S.; Wu, Z. (2015): Understanding Taxi Service Strategies From Taxi GPS Traces. In: Transactions on Intelligent Transportation Systems 16, 1, 123-135. doi: 10.1109/TITS.2014.2328231 